\title{
Lesion Revascularization Clinical Indicator
}

National Cancer Institute

\section{Source}

National Cancer Institute. Lesion Revascularization Clinical Indicator. NCI Thesaurus. Code C119556.

An indication as to whether an appropriate clinical context was present before lesion revascularization occurred. 